\title{
8.3 The structure of inter-level coordination of parameters for assessing the quality of public transport services
}

he leading role of urban public passenger transport (PPT) in ensuring the living conditions of cities and increasing its importance in shaping the socio-economic development of territorial environments has led to a set of approaches and methods for assessing the quality of public transport services (QPTS). The most widespread approaches are those in which the quality of transport services is understood as a set of properties of the transportation process and passenger transportation system, which determine the satisfaction of passengers' needs in travel in accordance with the established regulatory requirements [427-428]. Evaluation of QPTS is an important part of the study of the effectiveness of MGPT and usually involves a comparative procedure on a set of parameters to describe the state of the transport process [429433].

In studying the effectiveness of complex ergatic systems, which include PPT, the use of simple analytical methods of comparison leads to a number of inaccuracies and subjectivity due to the large size of the problem and the need to take into account a large number of heterogeneous factors inherent in PPT. Such an example is the procedure of quality assessment at the time of implementation of the movement [434437]. The inaccuracy of estimation when using travel time as a QPTS criterion is due to the randomness of motor operations on the network, and this leads to significant heterogeneity of the results. The difficulty of establishing a single standard (standard) of travel time is due to the individual requirements and views of certain categories of consumers of transport services.

Quality assessment (control) at the consumer level should be carried out by establishing the degree of compliance of the provided transport offer in relation to consumer requirements. Linguistic variables are used as basic degrees of QPTS compliance: excellent $\left(A_{v}\right)$, good $\left(A_{w}\right)$, satisfactory $\left(A_{a}\right)$, bad $\left(A_{b}\right)$, very bad $\left(A_{d}\right)$. The affiliation of the state of transport services to the degree of compliance is determined by the level of complaints received from consumers of transport services. 
A complaint arises when at least any parameter of the total number of transport service indicators $\left(n_{y c}\right)$ has a value that does not meet the established requirements of consumers. Determining the distribution of parameters by QPTS compliance is the main task of its identification. The solution of such a problem involves assigning the parameters of the object under study (route), which is set as a set of observations, to one of the mutually exclusive classes. This means that there is a unique mapping of the set of observations, which is a finite numerical set $\left\{N_{c p}^{m}\right\}$, to a set of classes of the degree of conformity QPTS $\left\{A_{i}\right\}, i=\overline{1, k_{q}}$, the total number $k_{q}$, which for the selected degrees of correspondence takes the following form: $\left\{N_{c p}^{m}\right\} \rightarrow\left\{A_{v}, A_{w}, A_{a}, A_{b}, A_{d}\right\}$. Depending on the completeness of information on the statistical characteristics of the parameters of transport services, the distribution procedure is implemented in order to establish the conditions for distinguishing the degrees of QPTS or assign rules for their recognition. At the stage of establishing the basic level of QPTS on the basis of selective monitoring for each element of PPT (route) the boundaries of the distribution of indicators by degrees of quality are determined. The defined rules of distribution of parameters are further used to recognize the state of QPTS which can be achieved by implementing a set of management measures.

The process by which the system gradually acquires the ability to respond to the required responses to certain sets of external and managerial influences is called learning. In the study of transport systems, it is implemented on the basis of modeling of real processes with subsequent processing of the results. Training is part of the classification process and has the ultimate goal of forming reference descriptions of QPTS degree classes. The form of reference descriptions is determined by the method of their use in the decisive rules established by the method of assigning the parameters of transport services achieved by implementing the management measure to any image of the degree of evaluation of QPTS. A training sample is required to construct the decisive rules. A training sample is a set of objects given by the values of features that belong to consumers of a particular type and are reliably known to the "teacher" and 
communicated to them by the learning system. The end result of training is to establish the limits of technological parameters that determine the degree of compliance of QPTS with consumer requirements.

The allocation of the boundaries of the transition between the levels of perception involves the procedure of interviewing passengers on specific routes on the basis of "indicator - the degree of compliance with QPTS." Negative assessments of QPTS are determined by the level of satisfaction of consumer requirements by the number of indicators $\left(n_{y c}\right)$ of the consumer level according to the selected indicators: waiting time, travel time, regularity of communication, the level of vehicle occupancy.

In the course of cluster analysis, boundaries are set for each transition of the degree of consumer quality, namely: from excellent to good $A_{v} \rightarrow A_{w}$, the limit is in $y_{c i}^{v}$, from good to satisfactory - $A_{w} \rightarrow A_{a}-y_{c i}^{w}$, from satisfactory to bad - $A_{a} \rightarrow A_{b}-$ $y_{c i}^{a}$, from bad to very bad $-A_{b} \rightarrow A_{d}-y_{c i}^{b}$. The clustering procedure is based on the processing of data obtained through questionnaires. The structure of the questionnaire contains fields for choosing the degree of subjective assessment of passengers' satisfaction with QPTS and a set of questions that determine the operational parameters of the trip.

Based on the degree of conformity of consumer quality, the actual state of the transport service system is determined. The quality of transport services PPT - is its characteristic at the appropriate time of operation, which is described by a set of stationary and dynamic technical and operational, social, resource and economic indicators. Table 1 presents typical quality conditions of the PPT transport service system. 
Characteristics of the states of PPT elements

\begin{tabular}{|l|c|c|}
\hline $\begin{array}{c}\text { Degree of consumer quality } \\
\text { assessment }\end{array}$ & $\begin{array}{c}\text { Range of change of } \\
\text { evaluation indicators }\end{array}$ & $\begin{array}{c}\text { The share of consumer } \\
\text { complaints }\end{array}$ \\
\hline excellent $\left(A_{v}\right)$ & $y_{c i}^{n} \geq y_{c i}^{v}, \forall i \in n_{y c}$ & 0 \\
\hline good $\left(A_{w}\right)$ & $y_{c i}^{v}>y_{c i}^{n} \geq y_{c i}^{w}, \forall i \in n_{y c}$ & $0<p_{u c}^{n} \leq p_{u c}^{w}$ \\
\hline satisfactorily $\left(A_{a}\right)$ & $y_{c i}^{w}>y_{c i}^{n} \geq y_{c i}^{a}, \forall i \in n_{y c}$ & $p_{u c}^{w}<p_{u c}^{n} \leq p_{u c}^{a}$ \\
\hline badly $\left(A_{b}\right)$ & $y_{c i}^{a}>y_{c i}^{n} \geq y_{c i}^{b}, \forall i \in n_{y c}$ & $p_{c u}^{a}<p_{c u}^{n} \leq p_{c u}^{b}$ \\
\hline very bad $\left(A_{d}\right)$ & $y_{c i}^{b}>y_{c i}^{n}, \forall i \in n_{y c}$ & $p_{u c i}^{b}<p_{u c}^{n}$ \\
\hline
\end{tabular}

When determining the degree of consumer quality to regulatory requirements, the compliance factor can take only two alternative values - 0 or 1 . If all service parameters meet their regulatory values, the compliance factor is 1 , and if at least one parameter does not meet -0 . The service can not meet the requirements of the standard, for example, half, ie half of the indicators it meets, and the other half - no. Therefore, if at least one indicator of the service does not meet, it is already non-standard and is not subject to its presentation as quality. The share of negative assessments provided by consumers is set during the solution of the problem of assessing the criterion of compliance with the parameters of the degree of QPTS

$$
p_{u c}^{\sum}=\frac{\sum_{i=1}^{n_{r p}}\left(1-z_{r p_{i}}^{b}\right)}{n_{r p}},
$$

where $z_{r p_{i}}^{b}$ - assessment of the quality of transport services by the respondent from the sample survey group;

$n_{r p}$ - the number of respondents in the survey sample. 
The assessment of each category of quality of transport service is determined based on the conditions of compliance with the limit of "excellent" degree QPTS

$$
z_{p p_{i}}^{b}=\left\{\begin{array}{l}
1, n p u y_{c i}^{n} \geq y_{c i}^{v} \\
0, n p u y_{c i}^{n}<y_{c i}^{v}
\end{array},\right.
$$

where $y_{c i}^{v}$ - the actual value of the transport service parameter;

$y_{c i}^{n}$ - the value of the parameter of transport service that corresponds to the limit of "excellent" degree of evaluation of QPTS.

The overall positive QPTS score for each respondent is set based on the presence of a complaint for any parameter

$$
z_{r p_{i}}^{b}=\left\{\begin{array}{l}
1, n p u z_{p p_{i}}^{b}=1, \forall i \in n_{y c}, \\
0
\end{array}\right.
$$

where $n_{y c}$ - the number of parameters for assessing the consumer quality of transport services.

In order to determine the correspondence of the distribution of the share of complaints to the QPTS levels, it is necessary to determine not only the general perception of the service, but also to establish the share of the parameters by which they arise

$$
p_{u c}^{n}=\frac{\sum_{i=1}^{n_{r p}} \sum_{j=1}^{n_{y c}}\left(1-z_{p p_{i j}}^{b}\right)}{n_{r p} \cdot n_{y c}},
$$

where $z_{p p_{i j}}^{b}$ - assessment of the quality of transport service of the respondent $i$ from the group of sample survey according to the parameter $j$. 
Inter-level consistency involves establishing the relationship between degrees for different levels of QPTS (technical, technological and consumer). The consumer level of QPTS is the basis for assessing the degree of compliance. However, the possibility of its change is determined not only by the consumer and organizational environment, but also by the state of functioning of transport infrastructure and transport enterprises. To implement the procedure of inter-level coherence, it is necessary to establish a connection: $Y_{p} \rightarrow A\left(Y_{c}\right), Y_{e} \rightarrow A\left(Y_{c}\right)$. The connection $Y_{p} \rightarrow A\left(Y_{c}\right)$ reproduces the change of QPTS states during the implementation of control measures within the transport enterprise (choice of brand and number of rolling stock, establishment of traffic on the route, distribution of rolling stock between routes, etc.), and communication $Y_{e} \rightarrow A\left(Y_{c}\right)$ - change of states during control measures within the transport infrastructure (establishment of the mode of movement on street, optimization of time of stay in stopping points, rationalization of placement of stopping points, etc.). A sequence of transitions $Y_{e} \rightarrow A\left(Y_{p}\right), Y_{p} \rightarrow A\left(Y_{e}\right)$ is used to establish a connection $Y_{e} \rightarrow A\left(Y_{c}\right) \rightarrow A\left(Y_{p}\right), Y_{p} \rightarrow A\left(Y_{c}\right) \rightarrow A\left(Y_{e}\right)$.

Parameterization of the selected connection requires the formation of appropriate transition matrices. Imagine in the system -dimensional topological diversity three different coordinate systems that differ in the number of parameters and measurement scales. Each coordinate system has its own character of space formation which does not allow them to intersect. The first coordinate system describes the technical level of QPTS and is a set of scales $\left\{y_{p g}, y_{p n}, y_{p t}, y_{p c i}, y_{p w}\right\}$, the second technological $\left\{y_{e t}, y_{e v}, y_{e h}, y_{e l}, y_{e r}\right\}$, the third - consumer $\left\{y_{c t}, y_{c p}, y_{c u}, y_{c e}\right\}$. The presented spaces may exist separately from each other, but there is a clear relationship between them, which is realized through a change in the actual state of the transport service system and its quality. The change of the actual state at one level leads to the combination of parameters of the transport process at another level. In fig. In Fig. 1 presents a graphical interpretation of the inter-level change of QPTS parameters. 


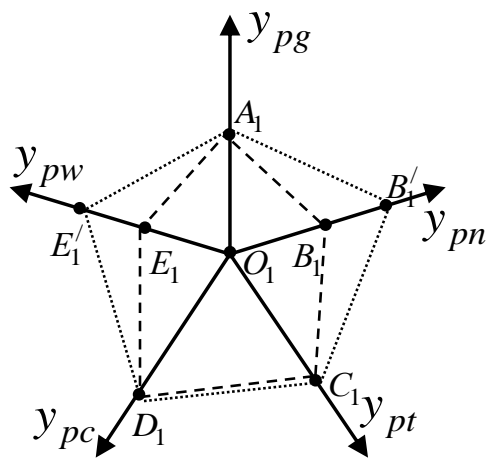

a)

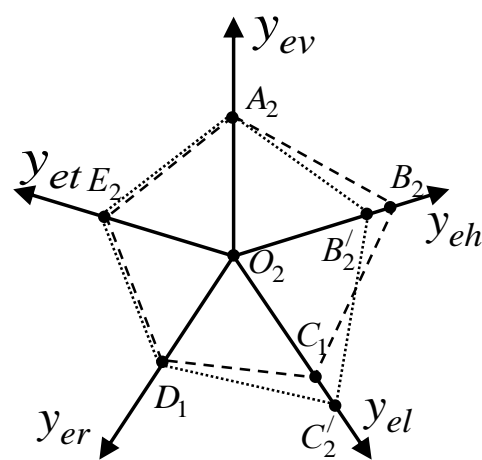

б)

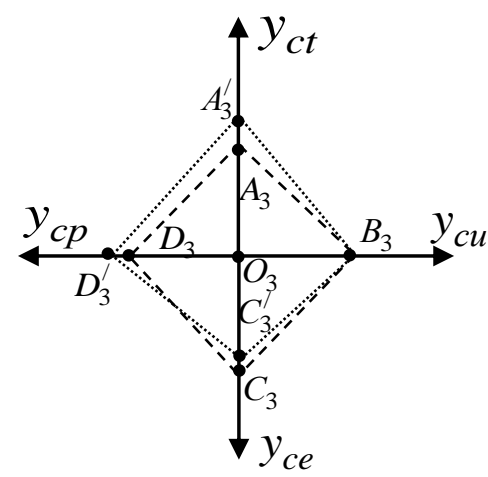

в)

$a$-technical environment; $\sigma$ - technological environment; $b$ - consumer and organizational environment.

Legend: $\because$ - basic state of parameters,

Figure 1. Graphical interpretation of the inter-level change of QPTS parameters

In fig. 1 shows the conditional coordinate systems of the parameters that characterize QPTS at different levels of its representation. As an example, consider the implementation of the control flow $z_{p n}$ within the transport company to increase the number of rolling stock on the route. During the implementation of such a measure there will be a change (improvement) of the basic state of the parameters of the technical level of QPTS: the number of vehicles $\left(y_{p n}\right)$ and the average level of filling of vehicles $\left(y_{p w}\right)$. The result of such a measure is to increase the technical level of QPTS, which is shown in the figure by changing the contour of the figure $A_{1} B_{1} C_{1} D_{1} E_{1} \rightarrow A_{1} B_{1}^{\prime} C_{1} D_{1} E_{1}^{\prime}$ and increasing its plane. However, this measure also leads to changes in the parameters of the technological and consumer level of QPTS and the corresponding contours of the figures $A_{2} B_{2} C_{2} D_{2} E_{2} \rightarrow A_{2} B_{2}^{\prime} C_{2}^{\prime} D_{2} E_{2}$, $A_{1} B_{1} C_{1} D_{1} \rightarrow A_{1}^{\prime} B_{1} C_{1}^{\prime} D_{1}^{\prime}$. Commenting on these changes, it should be noted their ambiguous nature of inter-level impact, which at some levels can lead to both an increase in QPTS parameters and a decrease. Thus, increasing the number of rolling stock on the route allows you to positively affect the interval of movement $\left(y_{e l}\right)$ and 
negatively - the downtime at stops $\left(y_{e h}\right)$. Increasing the number of vehicles without implementing measures to streamline their arrival at stops can lead to queues and additional unproductive downtime. In turn, unproductive downtime increases the cost of passengers' time for boarding and disembarking passengers $\left(y_{c e}\right)$, which is part of determining the degree of service QPTS. Although in general, when changing the contour of the figure $A_{1} B_{1} C_{1} D_{1} \rightarrow A_{1}^{\prime} B_{1} C_{1}^{\prime} D_{1}^{\prime}$, its plane increased (due to increasing parameters $y_{c t}$ and $y_{c p}$ ), but there was a deterioration of a particular indicator $y_{c e}$, which may lead to its going beyond the appropriate degree of assessment and as a result of passengers' complaints about QPTS. To formalize the inter-level transition of QPTS parameters, it is necessary to establish equivalence between the limits of the degree of consumer quality and the parameters of technical and technological parameters. 